\title{
Concomitant invasive pulmonary aspergillosis and Candida zeylanoides bloodstream infection in an acute myeloblastic leukemia patient
}

\author{
Ion Antohe ${ }^{1,2}$, Angela Dascalescu ${ }^{1,2}$, Georgiana Butura ${ }^{2}$, Amalia Merticariu $^{2}$, Catalin \\ Danaila $^{1,2}$, Karina Bilavski ${ }^{3}$, Mihaela Zlei ${ }^{4,5}$, Petru Cianga ${ }^{*, 5}$
}

${ }^{1}$ Haematology Department, "Grigore T. Popa" University of Medicine and Pharmacy, lasi, ${ }^{2}$ Haematology Department, Regional Oncology Institute, lasi, ${ }^{3}$ Morphopathology Department, Regional Oncology Institute, lasi, ${ }^{4}$ Laboratory Of Medical Investigations, Regional Oncology Institute, Iasi, ${ }^{5}$ Immunology Department, "Grigore T. Popa" University of Medicine and Pharmacy, lasi

\begin{abstract}
Current management of acute myeloblastic leukemia (AML) involves induction chemotherapy followed by risk stratified consolidation approaches, consisting of high dose chemotherapy or allogeneic stem cell transplantation. AML patients are at high risk of invasive fungal disease, particularly mold infections, due to the leukemia and chemotherapy related immune deficit. We present an AML patient with prolonged fever and neutropenia after induction therapy, during which she developed concomitant Candida zeylanoides bloodstream infection and invasive pulmonary aspergillosis. The response to Caspofungin and Voriconazole therapy was delayed, and this dictated the surgical management of the remnant pulmonary lesions. A histological confirmation of aspergillosis was thus obtained. Evaluation of host risk factors for invasive fungal disease, prompt scale-up of the diagnosis scheme and initiation of antifungal therapy are mandatory in order to ensure patient survival.
\end{abstract}

Keywords: Pulmonary Aspergillosis, Acute Myeloid Leukemia

\section{Introduction}

Acute myeloblastic leukemia (AML) is a highly aggressive proliferation of the immature myeloid precursor cells, whose optimal management depends upon timely administration of chemotherapy regimens and allogeneic stem cell transplantation availability. Disease and therapy related immune suppression result in a high risk of opportunistic infection. Since the introduction of Candida prophylaxis with Fluconazole, we have witnessed a shift in the spectrum of

Received: August 2015; Accepted after review: September 2015; Published: September 2015.

${ }^{*}$ Corresponding author: Prof. Dr. Petru Cianga, MD, PhD, Immunology Department, „Grigore T. Popa” University of Medicine and Pharmacy, 16 Universitatii str., lasi, Romania

E-mail: petrucianga@hotmail.com fungal infections in these patients, with mold infections and particularly Aspergillus fumigatus accounting for the majority of cases [1]. Invasive pulmonary aspergillosis causes important morbidity and mortality in AML patients and favors disease relapse by delaying therapeutic sequences and thus altering the dose intensity of the intended treatment regimen [2].

Diagnosing in due time an invasive pulmonary aspergillosis requires, besides a high clinical suspicion, the preemptive use of imagistic and serologic diagnostic tools and prompt initiation of the antifungal therapy. Voriconazole is considered the backbone treatment of invasive pulmonary aspergillosis [3]. 


\section{Case report}

We present the case of a 31 year-old pregnant female patient, admitted to the Hematology Clinic of the Regional Oncology Institute, lasi in February 2014 for marked fatigue and productive cough, experienced during the previous month. The blood count revealed pancytopenia with grade IV neutropenia and $35 \%$ blast cells on the peripheral blood smear. Bone marrow examination revealed extensive infiltration with myeloid blasts displaying an M5 immunophenotype (CD45-/+ low, CD117+, CD34+, CD13+, CD33+ low, HLA/DR+, CD38+ high, CD36-, CD64-/+ low (6\%), CD56+, CD123+, MPO-/+ (2\%), NG 2+). Molecular testing did not identify any specific gene fusion products. Karyotype examination was normal. The patient had no compatible HLA sibling.

A diagnosis of intermediate risk Acute Monoblastic Leukemia- not otherwise specified (NOS) was established according to the 2008 World Health Organization Classification of Tumors of Hematopoietic and Lymphoid Tissues and the patient was considered eligible for allogeneic stem cell transplantation from unrelated donor after achieving complete remission (CR). There is no evidence regarding the influence of pregnancy upon the evolution of acute leukemias [4]. On the other hand, as the patient was in the first trimester of pregnancy, a therapeutic abortion had to be performed, considering the potential adverse effects of chemotherapy upon the fetus.

A standard " $7+3$ " induction therapy (Cytarabine100-200 mg/m $\mathrm{m}^{2}$ continuous IV infusion for 7 days and Idarubicin 12 $\mathrm{mg} / \mathrm{m}^{2} /$ day for 3 days) was initiated, with immediate onset of profound leukopenia and neutropenia, with a total duration of 30 days. Taking into account the high risk of developing invasive fungal disease, we considered the patient eligible for Posaconazole prophylaxis. However, as Posaconazole was not available in our center at that moment, Fluconazole prophylaxis was performed instead.

On day 6 post induction the patient became febrile and presented X-ray features of left inferior lobe pneumonia. She immediately defervesced after empirical
Piperacilin plus Tazobactam and Gentamicin therapy (providing broad spectrum coverage against Gram positive and Gram negative bacteria, including Pseudomonas aeruginosa) was initiated, but on day 19 post induction the fever relapsed, without any other further clinical evidence of infection.

The antibiotic regimen was switched to Meropenem (targeting Gram negative bacteria, especially extended spectrum betalactamase producing microorganisms) and Teicoplanin (targeting Gram positive bacteria and especially meticillin resistant staphylococci), but without resolution of the febrile syndrome over the next 48 hours. On day 21 the blood cultures became positive for Candida zeylanoides, so that broad-spectrum antibiotherapy was stopped, Fluconazole prophylaxis was discontinued and intravenous Caspofungin was added. Despite that, the fever persisted and we noted the onset of respiratory symptoms (severe cough, anterior thoracic pain, and dyspnea) on day 30 post induction.

A CT examination of the thorax revealed a consolidation syndrome in the right superior lobe, a paracardiac abscess in the right middle lobe and an infiltrative nodule in the right inferior lobe (Figure 1, Figure 2). Serum Galactomannan antigen (a component of the Aspergillus cell wall), routinely investigated in neutropenic patients with high suspicion of invasive fungal disease, was negative at this moment.

Even though specific diagnostic methods (such as PCR for the ITS2 (internal transcribed spacer 2) sequences $[5,6]$ were not available, taking into account the patient's risk factors and the imagistic data according to the 2008 EORTC criteria (see supplementary material), we considered the possibility of invasive pulmonary aspergillosis and we empirically initiated the therapy with Voriconazole. The patient defervesced within 24 hours. Neutrophil and platelet recovery occurred on day 32 post induction, allowing us to perform a broncho-alveolar lavage (BAL). Galactomannan testing and Aspergillus spp. cultures from the BAL fluid were positive, allowing us to consider the diagnosis of probable invasive pulmonary aspergillosis. Progressive improvement of the clinical status 
permitted the discharge of the patient on day 41 post induction. Complete remission was confirmed at this moment by morphological and flow cytometrical bone marrow
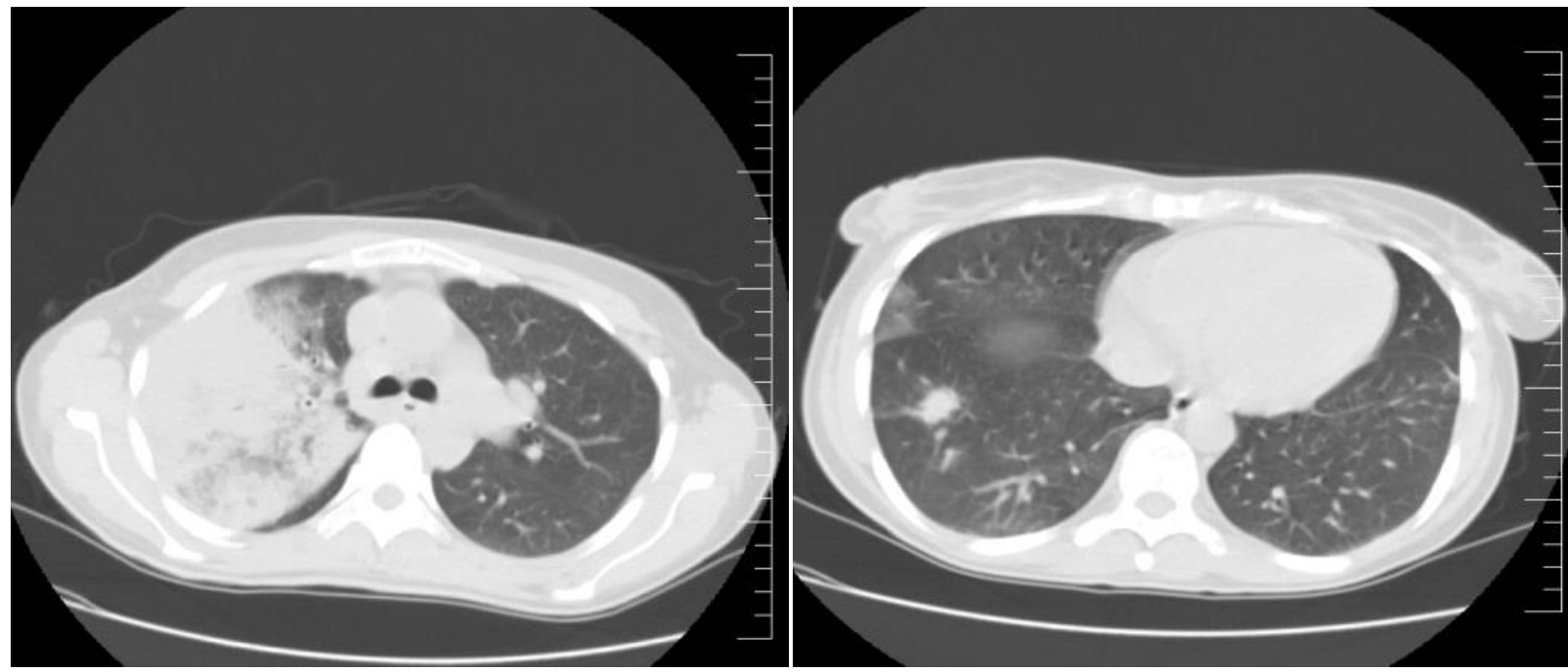

Fig. 1. Extensive infiltrates in the right superior lobe (Day 30 post induction).

The patient was readmitted on day 69 post induction with residual persistent cough. A thoracic CT scan after 38 days of Voriconazole therapy showed marked regression of the upper lobe infiltrates, but progression of the right inferior lobe lesion. Under these circumstances, the patient required a surgical approach, so that an atypical inferior lobe examination. Intravenous Caspofungin was discontinued and the patient remained on oral Voriconazole.
Fig. 2. Infiltrative nodule with discrete halo sign in the lateral segment of the right inferior lobe (Day 30 post induction).

resection was performed on day 88 post induction.

The pathology examination revealed the presence of narrow tubular septate hyphae, branching at angles of $45^{\circ}$ in all the examined sections, supporting the diagnosis of invasive pulmonary aspergillosis (Figure 3). No angioinvasive features were present.

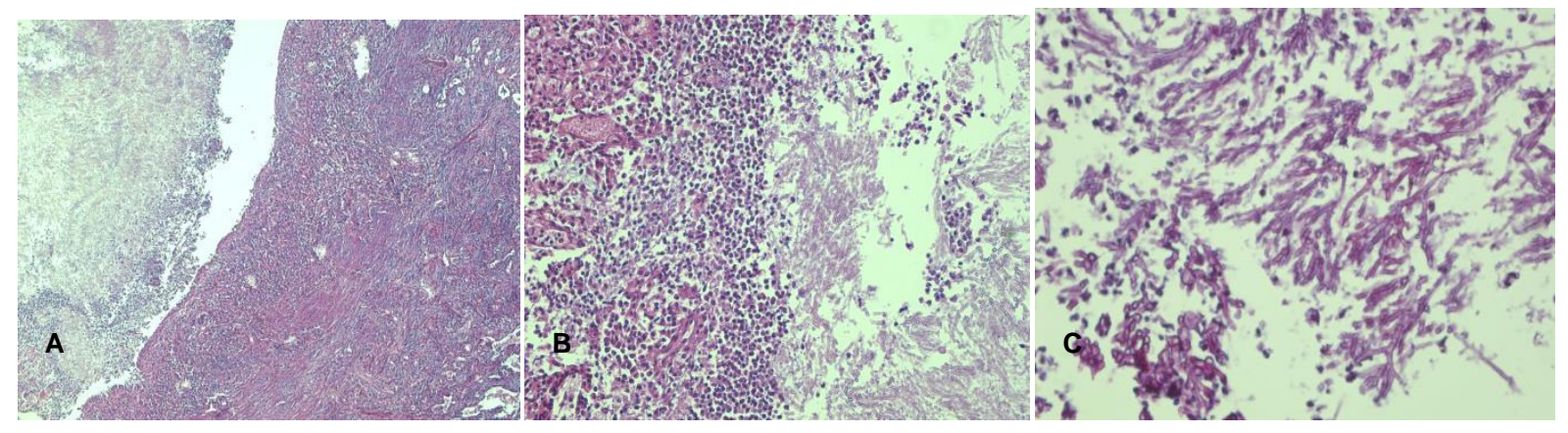

Fig. 3. Pathology examination reveals presence of Aspergillus hyphae in all examined sections. A: Fragment of the pulmonary tissue displaying a central cavity encircled by a granulation tissue with chronic inflammatory infiltrate. In the cavity lumen multiple colonies of hyphae are present ("fungus ball" appearance). (HE, x5) B: granulation tissue surrounding hyphal elements. (HE, x20) C: tubular narrow (4-6 $\mu \mathrm{m})$ monomorphic hyphae, regularly septate, branching at angles of $45^{\circ}$. Both viable (basophilic) and necrotic (eosinophil) hyphae were present. (PAS, x40) 
The evolution after surgery was favorable and the patient continued the oral Voriconazole therapy for a total of 5 months.
Repeated chest CT examinations revealed a complete resolution of the pulmonary infiltrates (Figure 4).

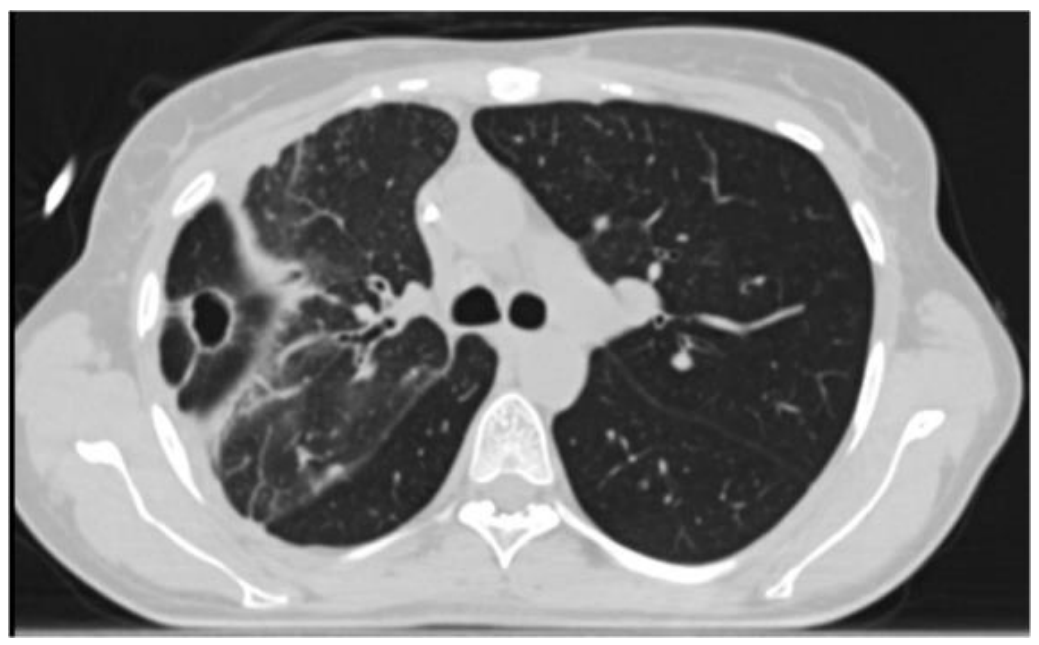

Fig. 4. Resolution of right upper lobe infiltrates (Day 90 post induction). Minimal post surgery encapsulated pneumothorax is present in the lateral segment of the middle lobe

Consolidation chemotherapy, consisting of two cycles of high dose Cytarabine, was administered starting day 94 post induction. Taking into account the preexisting fungal infection, secondary Voriconazole prophylaxis was employed.

The patient suffered leukemia relapse 10 months after diagnosis. We have opted for IdaFLAG (Idarubicine, Fludarabine, high dose Cytarabine, followed by Filgrastim administration) as a reinduction regimen, obtaining a second complete remission. Secondary Posaconazole prophylaxis was used during this period, as the patient developed a severe sun-burn-like skin reaction after oral Voriconazole. No other fungal related manifestations could be noticed. The patient is currently in complete remission, awaiting an allogeneic stem cell transplantation from an unrelated donor.

\section{Discussions}

We present the case of a young female patient with intermediate risk AML, eligible for allogeneic stem cell transplantation from an unrelated donor, for which severe infectious complications prevented the administration of consolidation chemotherapy for more than 90 days, thus favoring leukemia relapse. Despite combined Caspofungin and Voriconazole therapy, the delayed response ( $>30$ days) of the fungal pulmonary lesions imposed surgical management.

We have considered this patient to be at high risk for developing invasive fungal disease according to the following criteria: the induction treatment for AML, history of use of broad spectrum antibiotics and history of prolonged neutropenia (> 10 days).

On day 6 post induction therapy a broad spectrum antibiotic therapy was initiated and while the initial response was favorable, the fever relapsed and persisted despite the Candida zeylanoides targeted Caspofungin treatment.

Furthermore, multiple other diagnosis issues had to be considered. We could not rule out completely the possibility of a bacterial infection, even though the fever had not responded to the Meropenem and Teicoplanin combination. Given the normal transthoracic echocardiography, a bacterial or fungal endocarditis was considered less probable, even if its negative predictive value is not $100 \%$. The co-existence of an alternative invasive fungal infection, particularly pulmonary aspergillosis, was also taken into account and we have thus performed a chest 
CT that raised indeed a high suspicion of invasive pulmonary aspergillosis in this case. Under circumstances such as these it is highly recommended to enhance the diagnostic workup by including imaging tests, serial serum Galactomannan assessment and, whenever possible, Galactomannan testing and cultures from BAL fluid, rather than relying solely on empirical broad spectrum antifungal therapy. Histological confirmation following lung biopsy is considered ideal; however, severe thrombocytopenia often prevents this approach.

Scaling-up the diagnostic approach in due time for febrile neutropenic patients is essential for the successful management, especially in patients suspected of invasive pulmonary aspergillosis [7]. It is known that the transition from the bronchoalveolar to the angioinvasive phase of the infection evolves in a very narrow timeframe of just several hours in neutropenic patients [8]. Prompt preemptive initiation of antifungal treatment is thus vital.

When facing high risk neutropenic patients with persistent fever, for which invasive fungal disease is ruled out, it is a common approach in our center to test for pulmonary tuberculosis and even administrate empiric tuberculostatic therapy. Finally, in patients with persistent febrile syndrome, bone marrow examination should be considered to exclude refractory disease. It is questionable whether primary Posaconazole prophylaxis would have been able to prevent the Aspergillus infection, allowing for timely consolidation

\section{References}

1. van Burik $\mathrm{JH}$, Leisenring $\mathrm{W}$, Myerson $\mathrm{D}$, et al. The Effect of Prophylactic Fluconazole on the Clinical Spectrum of Fungal Diseases in Bone Marrow Transplant Recipients with Special Attention to Hepatic Candidiasis. An Autopsy Study of 355 Patients. Medicine 1998; 77(4):246-254.

2. Lin SJ, Schranz J, Teutsch SM. Aspergillosis Case-Fatality Rate: Systematic Review of the Literature. Clin Infect Dis 2001; 32:358-366.

3. Rüping MJ, Vehreschild JJ, Cornely OA. Antifungal Treatment Strategies in High Risk Patients. Mycoses 2008; 51(Suppl 2):46-51. chemotherapy. However, it is known that Posaconazole prophylaxis reduces the incidence of invasive fungal disease from $8 \%$ to $2 \%$ in high risk $A M L$ and myelodysplastic syndrome patients [9-11]. At least part of its superiority in prophylaxis over other antifungal azoles, like Fluconazole, appears to be due to its hydrophobic nature and due to the preferential concentration (up to 400 fold) in the membrane compartments of alveolar macrophages and epithelial cells [12].

\section{Conclusions}

We present a rare case of concomitant pulmonary invasive aspergillosis and Candida zeylanoides bloodstream infection. The diagnosis and management of invasive pulmonary aspergillosis in neutropenic patients is difficult and challenging, requiring careful consideration of the patients' risk factors, dynamical clinical assessment and early preemptive use of paraclinical tools and antifungals.

\section{Acknowledgements}

This paper benefited from financial support from the project "CERO- Profil de carieră: cercetător român”, contract no. POSDRU/159/1.5/S/135760, co-financed from the European Social Fund via the Sectorial Operational Program for Human Resource Development 2007-2013.

4. Chelgoum Y, Vey N, Raffoux E, et al. Acute Leukemia during pregnancy. A report on 37 patients and review of the literature. Cancer 2005; 104:110-117.

5. Liu P, Li B, Yin R, et al. Development and evaluation of ITS- and aflP-based LAMP assays for rapid detection of Aspergillus flavus in food samples. Can J Microbiol. 2014; 60(9):579-84.

6. Babouee Flury B, Weisser M, Prince Savic S, et al. Performances of two different panfungal PCRs to detect mould DNA in formalin-fixed paraffin-embedded tissue: what are the limiting 
factors? BMC Infectious Diseases, 2014; 14:692.

7. Liss B, Vehreschild JJ, Bangard C, et al. Our 2015 approach to invasive pulmonary aspergillosis. Mycoses 2015; 58:375-382.

8. Nucci M, Nouér SA, Cappone D, et al. Early Diagnosis Of Invasive Pulmonary Aspergillosis In Hematologic Patients: An Opportunity To Improve The Outcome. Haematologica 2013; 98:1657-1660.

9. Vehreschild JJ, Ruping MJ, Wisplinghoff $\mathrm{H}$, et al. Clinical Effectiveness of Posaconazole Prophylaxis in Patients with Acute Myelogenous Leukaemia (AML): a 6 year Experience of the Cologne AML Cohort. $J$ Antimicrob Chemother 2010; 65(7):1466-1471.
10. Cornely OA, Maertens J, Winston DJ, et al. Posaconazole vs. Fluconazole or Itraconazole Prophylaxis in Patients with Neutropenia. $N$ Engl J Med 2007; 356(4):348-359.

11. Girmenia C, Frustaci AM, Gentile G, et al. Posaconazole Prophylaxis during front-line Chemotherapy of Acute Myeloid Leukemia: a single-center, real-life experience. Haematologica 2012; 97(4):560-567.

12. Sheppard DC, Campoli $P$, Duarte RF. Understanding Antifungal Prophylaxis with Posaconazole in Hematology Patients: an evolving bedside to bench story. Haematologica 2014; 99(4):603-604. 


\section{Supplimentary Materials}

TABLE 1. EORTC criteria for proven lower respiratory tract invasive mold infection

\begin{tabular}{|c|c|}
\hline Analysis/specimen & \\
\hline Microscopic analysis & $\begin{array}{l}\text { Histopathologic, cytopathologic, or direct microscopic } \\
\text { examination of a specimen obtained from a normally sterile site }\end{array}$ \\
\hline Culture: sterile material & $\begin{array}{l}\text { Recovery of a mold by culture of a specimen obtained by a } \\
\text { sterile procedure from a normally sterile and clinically or } \\
\text { radiologically abnormal site consistent with an infectious } \\
\text { disease process (excluding bronchoalveolar lavage fluid) }\end{array}$ \\
\hline Culture: blood & Blood culture that yields a mold \\
\hline
\end{tabular}

TABLE 2. EORTC criteria for probable lower respiratory tract invasive fungal disease

\begin{tabular}{|c|c|}
\hline Host criteria & $\begin{array}{l}\text { Recent history of neutropenia }\left(<0.5 \times 10^{9} \text { neutrophils } / L \text { or }<500 \text { neutrophils } / \mathrm{mm}^{3} \text { ] for }>10\right. \\
\text { days) } \\
\text { Allogeneic stem cell transplant recipient } \\
\text { Prolonged corticosteroid therapy } \\
\text { Treatment with nucleoside analogues and T cell immunosuppressants during the past } 90 \text { days } \\
\text { Inherited severe immunodeficiency }\end{array}$ \\
\hline Clinical criteria & 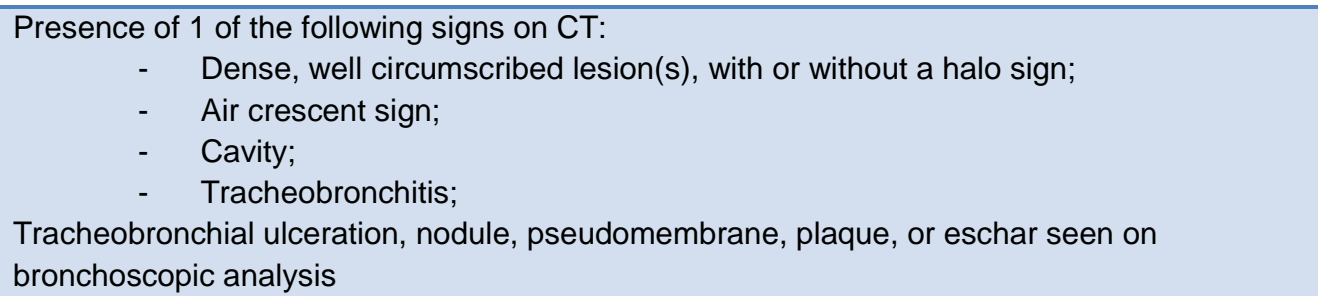 \\
\hline $\begin{array}{l}\text { Mycological } \\
\text { criteria }\end{array}$ & 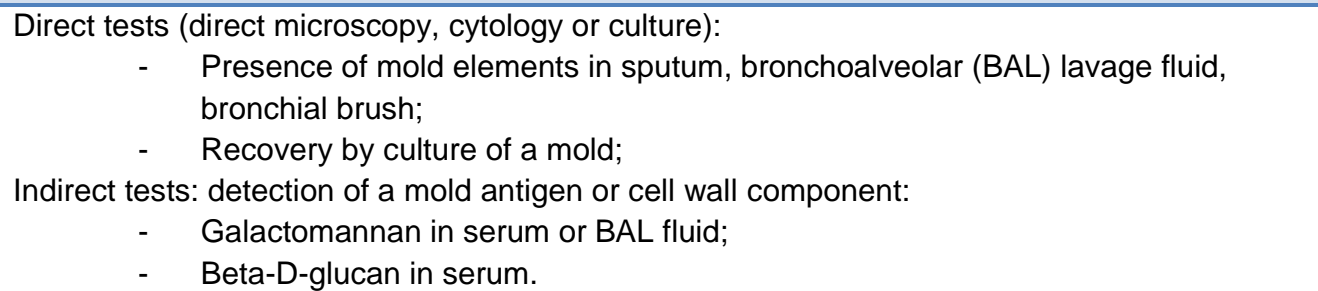 \\
\hline
\end{tabular}

